\title{
A profile of psychological maladjustment among school children in Sri Lanka
}

\author{
Piyanjali de Zoysa ${ }^{1 *}$, P A Newcombe ${ }^{2}$, L Rajapakse ${ }^{3}$ \\ 1. Department of Psychological Medicine, Faculty of Medicine, University of Colombo, Colombo 08
}

\begin{abstract}
This study examined the profile of psychological maladjustment among 12-year-old Sinhala speaking children in the Colombo district of Sri Lanka. The children ( $\mathrm{N}=1226)$ were selected from government schools in the Colombo district, using a stratified random sampling technique. The Personality Assessment Questionnaire, a self-administered instrument shown to have good psychometric properties, was adapted and validated to the Sri Lankan context and was then used in the study survey. Nearly half of the children in the study were psychologically maladjusted. Among the seven personality dimensions in the instrument that assessed psychological maladjustment, a majority of children had a deficit in the personality dimensions of independence and emotional stability. The paper discusses the possible reasons for these findings.
\end{abstract}

\section{INTRODUCTION}

Children, being the future decision-makers of a country, are an important national resource. Psychologically adjusted children would therefore be an asset to a nation. Hence, culture-specific information on the profile of psychological maladjustment among children would be useful when designing preventative and rehabilitation programs to reduce and overcome such maladjustment. Such information is scarce in Sri Lanka. This study attempted to bridge this gap by exploring the profile of psychological maladjustment among 12-year-olds attending government schools in the Colombo district of the country.

\section{METHOD}

This study was a part of a larger study on parent-child violence (De Zoysa, 2006). A sample of 1226 (60.8\% girls) 12 -year-olds was chosen by a stratified random sampling technique from all Sinhala language government schools in the Colombo district of Sri Lanka. Buddhists were a majority at $84.8 \%$ (6.4\% Islam; $4.1 \%$ Roman Catholic; 3.4\% Christian; 1.2\% Hindu) and 90.2\% were of Sinhalese ethnic origin (1.8\% Moor; 2.4\% Sri Lankan Tamil; 0.6\% Malay; 0.6\% Burgher; 0.4\% Indian Tamil; $0.1 \%$ Other). Permission for the study was obtained from the Ministry of Education and ethical approval from the Faculty of Medicine, Colombo. Verbal consent to take part in the study was obtained.

The Sinhala version of the Personality Assessment Questionnaire (Rohner, 1999) has shown good psychometric properties (PAQ: De Zoysa, Rajapakse, Newcombe, 2007) was used to determine the extent of psychological maladjustment in the children. The PAQ is a self-administered 42-item instrument designed to assess a child's perception of his/her self with respect to seven personality dimensions (De Zoysa, Rajapakse, Newcombe, 2007; Rohner, 1999). These are: hostility and aggression (e.g., "I think about fighting or being mean"), dependency (e.g., "I like my parents to give me a lot of love"), negative self-esteem (e.g., "I get unhappy with myself"), negative self-adequacy (e.g., "I feel I can do the things I want as well as most people"), emotional unresponsiveness (e.g., "I have trouble showing people how I feel"), emotional instability (e.g., "I feel bad or 
get angry when I try to do something and cannot do it"), and negative world view (e.g., "I feel that life is nice"). By summing the scores of these seven personality dimension scales, an overall score/assessment of the child's level of psychological maladjustment can be made.

Following the school principal's permission, the instrument was administered to all 12-year-olds who attended class on the testing day. Children were informed of the nature of the study and that confidentiality of answers would be maintained. The first author administered the instruments and was available throughout to clarify any doubts.

\section{RESULTS}

Of the sample of 1226 , only 4 children did not return completed PAQs. The PAQ scale scores were used to determine maladjustment for each of the personality dimensions (De Zoysa, 2006): 20\% of children were hostile and aggressive, 92\% were dependent, $15 \%$ had negative self-esteem, $16 \%$ felt inadequate, $12 \%$ was emotionally unresponsive, $56 \%$ were emotionally unstable, and $19 \%$ had a negative world-view. On the overall PAQ, 49.4\% were classified as psychologically maladjusted.

A series of analyses compared the seven personality dimensions across the variables of: (i) number of siblings; (ii) child's religion; (iii) child's gender, and (iv) grading of the child's school. For these analyses, the variable of religion was re-categorised as non-Buddhist and Buddhist to facilitate the analyses as the distribution of cases across all reported religions exceeded the cell ratio of 1:3 considered appropriate for between-groups analysis (Tabachnick \& Fidell, 1996).

Psychological maladjustment differed significantly with the number of siblings in the family. Children with three or more siblings reported significantly greater negative self-esteem and negative self-adequacy than those with fewer than three siblings. Children with three or more siblings reported significantly greater emotional unresponsiveness than children with no siblings.

Psychological maladjustment differed significantly by child's religion, with non-Buddhist children reporting significantly greater hostility and aggression, negative self-esteem, negative self-adequacy, emotional unresponsiveness and emotional instability than did the Buddhist children.
Psychological maladjustment also differed significantly by child's gender, where males reported significantly greater hostility and aggression while females reported significantly greater dependency and emotional instability.

Sri Lankan schools are categorised according to the resources in the school (Grade 1 schools have best resources, Grade 2 have average resources, Grade 3 have few resources and Grade 4 schools have minimal resources). Children in Grade 4 schools were not a part of this study as these schools only had primary classes (i.e. children below 11 years). Psychological maladjustment differed significantly with the resources in the school. Children in Grade 1 schools reported significantly less hostility and aggression, greater dependency, less negative self-esteem, and less negative self-adequacy than children in either Grade 2 or 3 schools. Further, children in Grade 1 schools reported significantly less emotional unresponsiveness than children in Grade 3 schools. Hence, as compared to children in Grade 2 and 3 schools, those in Grade 1 schools were more dependent though the latter were superior in all other personality dimensions.

\section{DISCUSSION}

In this study, $49.4 \%$ of the children were found to be psychologically maladjusted. This finding is corroborated by another study on Sri Lankan 12-year-olds in the Gampaha district of Sri Lanka where 44\% of the children were evaluated as psychologically maladjusted (De Zoysa, Rajapakse \& Newcombe, 2004). The finding that nearly half of the sample studied was psychologically maladjusted is noteworthy. Literature shows that such adjustment problems are associated with a variety of interpersonal, emotional and career difficulties in later life (Ladd, 1990). The instrument that measured psychological maladjustment, the PAQ, has shown good psychometric properties - validity and reliability - both internationally (Rohner, 1999) as well as in Sri Lanka (De Zoysa, Rajapakse and Newcombe, 2007). Hence, the results reported have been obtained from an instrument that is cross-culturally adapted, valid and reliable. Future studies on other aspects of validity and reliability of the PAQ, if undertaken, would strengthen its usefulness as an instrument. Hence, these studies would strengthen the authenticity of the results of psychological maladjustment reported here. 
With regards to the seven individual personality dimensions comprising psychological maladjustment, a majority of children had difficulty in being independent. Independence is a key requisite in a young person as it provides an important foundation for taking on adult roles with responsibility. It is possible that the Sri Lankan socialisation process, which is highly protective of its children and does not offer much personal freedom, may be one of the reasons that has led to this situation (De Zoysa, Rajapakse \& Newcombe, 2004). In the dimension of emotional instability, again, a majority of children showed an inability to manage their emotions appropriately. Emotional stability is a necessity for the initiation and development of healthy interpersonal relationships, both in childhood and in later years. Deficits in the Sri Lankan socialisation process, failings of its education system and absence of appropriate role models for children in the family, school, community, and in the wider social and political context may be some of the factors that have contributed to this finding. In terms of the remaining five personality dimensions of hostility and aggression, negative self-esteem, negative self-adequacy, emotional unresponsiveness, and negative world view, only a minority of the children indicated an unhealthy level. However, a note of caution is worthy here as, unlike the findings on the extent of overall psychological maladjustment, the findings on the seven personality dimensions need to be interpreted carefully as some of the scale internal consistencies were below the minimum accepted criteria (De Zoysa, Rajapakse \& Newcombe, 2007).

The present findings could be compared to data from 220 American nine-to-eleven year olds (Rohner, 1999). The Sri Lankan children showed significantly lower levels of hostility and aggression, negative self-esteem, negative self-adequacy, emotional unresponsiveness, negative emotional instability and negative world view and significantly a higher level of dependency than did the American children - that is, overall, stronger psychological adjustment. Hence, as compared to the American upbringing, certain familial, school, social and cultural influences in the Sri Lankan socialisation process may protect the psychological health of our children better, though the Sri Lankan rates of psychological maladjustment are, on its own, still high.

Results showed that an increase in family size was associated with a decrease in the children's positive evaluation of self (i.e. the personality dimensions of selfesteem and self-adequacy). Among other factors, such an increase in family size may deteriorate the parent-child quality time, whereby children may not be exposed to sufficient parental affirmations on their adequacy in life challenges. Such affirmations are important in developing children's evaluation of self. Factors such as sibling rivalry and parental comparison of one child with his/her sibling(s) may also deteriorate a child's positive evaluation of oneself. Results also showed that children with three or more siblings were more emotionally unresponsive than children with no siblings. Such children may have difficulty in being open or emotionally expressive. It is hypothesised that this crucial family size of three or more siblings, with its potential for parental role stress, may lead parents to adopt increasingly unhealthy parenting strategies that are associated with negative psychological characteristics in children.

Buddhist children reported significantly less hostility and aggression and greater self-esteem, self-adequacy, emotional responsiveness and emotional stability than did the non-Buddhist children. Among other factors, the socialisation process adopted at home may differ from one religion to another, which in turn may influence the level of adjustment of a given child. However, this result should be considered with caution as the number of cases for non-Buddhist children, though within statistically acceptable numbers, was far fewer than Buddhist children. Further research, with a more representative sample, may explore this preliminary finding further.

Boys were significantly more aggressive and hostile than girls, a finding that is in keeping with the gender differences in life span development in hostility and aggression. They were also more independent and emotionally stable than the girls. In a patriarchal society such as Sri Lanka, that encourages docility in their females, it is to be expected that girls would be more dependent. However, and contrary to expectations, girls reported more emotional instability than boys. There were no significant gender differences in the personality dimensions of self-esteem, self-adequacy, emotional responsiveness or negative world view.

Children of Grade 1 schools reported significantly higher self-esteem, higher self-adequacy, higher emotional responsiveness, lower hostility and aggression and greater dependency than children from Grade 2 or 3 schools. Attending a Grade 1 school, with greater academic and extra-curricular opportunities, and the pride of attending such a school (in Sri Lanka, the name of one's school is sometimes considered a symbol of prestige) may develop a greater sense of esteem and adequacy in these children. In the larger study in which this study was embedded, the study results showed that the parents of Grade 1 schools, as compared to parents of Grade 2 
or 3 schools were more nurturant (De Zoysa, 2006). In this larger study, these children also reported that they were less exposed to corporal punishment, domestic violence, school violence and community violence. Hence, this more nurturant parenting and less exposure to violence may have also contributed to the children of Grade 1 school's superior evaluation of themselves and less hostility and aggression, than children of Grade 2 or 3 schools. The generally poorer neighbourhoods of children attending Grade 3 schools may necessitate self-reliance and independence whereas children of Grade 1 schools may have been actively encouraged to be dependent. The children of Grade 1 schools' greater emotional responsiveness may be due to their parents and school encouraging emotional expression. Such parents/schools may have greater access to information on the importance of emotional expression in personality development, as compared to the limited information on such matters that parents/teachers of Grade 2 or 3 schools may have.

These findings have implications for educating Sri Lankan parents on child rearing. For instance, informing parents with three or more children about the stress of parenting many children and about the necessity to equip oneself with healthy parenting strategies to manage such a situation is essential. These findings also have implications for those in the education system and for those who work on social policies aimed at developing the psychological health of the Sri Lankan child. Strengthening and widening the geographical extent of the prevailing school counselling system to manage children with psychological problems and educating teachers, parents and the larger public on how children could be assisted to develop into psychologically healthy persons are some of the areas that could be inspired to improve based on these research findings.

\section{References}

1. De Zoysa, P., 2006. Parental use of physical force towards school children in the Colombo district: Prevalence, psychosocial correlates \& psychological consequences. Ph.D. University of Colombo, Sri Lanka, pp. 160 - 91.

2. De Zoysa, P., Rajapakse, L. \& Newcombe, P. A., 2004. A personality profile of Sinhala speaking school children in Sri Lanka. Proceedings of the 60th Annual Conference of the Sri Lanka Association for the Advancement of Science. Colombo, Sri Lanka.

3. De Zoysa, P., Rajapakse, L. \& Newcombe, P. A., 2007. Adaptation and validation of the Personality Assessment Questionnaire on 12-year-old children in Sri Lanka. In: L. S. Boyar, ed. New psychological tests and testing research. New York: Nova Science Publishers Inc, pp. 955-80.

4. Ladd, G. W., 1990. Having friends, keeping friends, making friends, and being liked by peers in the classroom: predictors of children's early school adjustment? Child Development, 61 (4), pp. 1081100.

5. Rohner, R. P., 1999. Handbook for the study of parental acceptance and rejection. USA: Rohner Research.

6. Tabachnick, B. G. \& Fidell, L. S., 1996. Using multivariate statistics. 3rd ed. New York: Harper Collins. 